\title{
Adult validation of a self-administered tablet audiometer
}

\author{
Mark Bastianelli ${ }^{1,2,3,4^{*}}$ (D, Amy E. Mark ${ }^{5,6}$, Arran McAfee ${ }^{1,2,3}$, David Schramm ${ }^{1,2,3,4,5,7}$, Renée Lefrançois ${ }^{6}$ and \\ Matthew Bromwich 2,3,4,5,6
}

\begin{abstract}
Background: There is evidence to suggest that rates of hearing loss are increasing more rapidly than the capacity of traditional audiometry resources for screening. A novel innovation in tablet, self-administered portable audiometry has been proposed as a solution to this discordance. The primary objective of this study was to validate a tablet audiometer with adult patients in a clinical setting. Secondarily, word recognition with a tablet audiometer was compared against conventional audiometry.
\end{abstract}

Methods: Three distinct prospective adult cohorts underwent testing. In group 1 and group 2 testing with the automated tablet audiometer was compared to standard sound booth audiometry. In Group 1, participants' pure tone thresholds were measured with an automated tablet audiometer in a quiet clinic exam room. In Group 2, participants completed monosyllabic word recognition testing using the NU-6 word lists. In Group 3, internal reliability was tested by having participants perform two automated tablet audiometric evaluation in sequence.

Results: Group 1 included 40 patients mean age was $54.7 \pm 18.4$ years old and 60\% female; Group 2 included 44 participants mean age was $55.2 \pm 14.8$ years old and $68.2 \%$ female; Group 3 included 40 participants with mean age of 39.4 + 15.9 years old and 60.5\% female. In Group 1, compared to standard audiometry, $95.7 \%$ (95\% Cl: $92.6-$ 98.9\%) of thresholds were within $10 \mathrm{~dB}$. In Group 2, comparing word recognition results, $96.2 \%$ (95\% Cl: 89.5-98.7\%) were clinically equivalent and within a critical difference range. In Group 3, One-way Intraclass Correlation for agreement for the both left- and right-ear pure tone average was 0.98. The mean difference between repeat assessments was $0(S D=2.1)$ in the left ear, and $0.1(S D=1.1)$ in the right ear.

Conclusion: Puretone audiometry and word recognition testing appears valid when performed by non-healthcare experts using a tablet audiometer outside a sound booth in a quiet environment.

Trial registration: ClinicalTrials.gov Identifier: NCT02761798.

Registered April, 2016 < https://clinicaltrials.gov/ct2/show/NCT02761798>

Keywords: Automated audiometry, Audiology, Tablet audiometry, Screening audiometry, Hearing loss

\section{Background}

In the 2016 report on hearing loss based on the Canadian Health Measures Survey audiometry results from 2012 to 2015 indicated that $40 \%$ of Canadian adults between 20 and 79 years of age had at least mild hearing loss in at least one ear [1]. Furthermore, $77 \%$ of these adults with measured hearing loss were unaware of their

\footnotetext{
* Correspondence: mark.bastianelli@gmail.com

${ }^{1}$ Faculty of Medicine, University of Ottawa, Ottawa, Ontario, Canada

${ }^{2}$ Department of Otolaryngology - Head and Neck Surgery, The Ottawa

Hospital, Ottawa, Ontario, Canada

Full list of author information is available at the end of the article
}

pre-existing diagnosis. With aging being the number one cause of hearing loss, the incidence of hearing loss is projected to rise dramatically. The number of adults aged 65 and over in Canada is expected to double to 9.9-10.9 million people by 2036 [2]. This poses a significant challenge in order to provide both timely and costeffective access for these patients to audiometric services.

It is well established that undiagnosed/untreated hearing loss can lead to physiological changes associated with auditory deprivation, as well as psychosocial changes of social isolation and depression $[3,4]$. Hearing

(c) The Author(s). 2019 Open Access This article is distributed under the terms of the Creative Commons Attribution 4.0 International License (http://creativecommons.org/licenses/by/4.0/), which permits unrestricted use, distribution, and 
loss can result in emotional, physical, cognitive, and behavioral consequences including impaired activities of daily living, decline in independence and reduced quality of life [5-8]. Early identification can help to reduce these deleterious effects, and lead to easier adjustment to hearing aid use $[9,10]$.

Conventionally, audiometric testing for adults is performed by a trained audiologist in a sound-insulated booth. However access to hearing healthcare has been shown to be limited based on several factors including geographic location, provider variables (access to specialist or primary care services) and socioeconomic status [11, 12]. Current guidance from the American SpeechLanguage-Hearing Association (ASHA) recommends that adults be screened every 10 years through from age 19-50 and every 3 years thereafter and yearly in patients with identified hearing loss [13]. In one study where 4556 American adults were surveyed, $65 \%$ of respondents reported having a hearing test greater than 10 years ago or never tested at all [14]. This emphasizes the crucial need for improvement of the current model for hearing health care in North America in order to provide patients with both timely as well as effective audiometric screening.

One emerging solution to this public health problem is to utilize automated audiometry for screening when conventional audiometry is not available. Automated audiometry uses a computer-based software as well as the standard protocols that are used by audiologists to perform both air and bone conduction hearing testing. Several reports have previously demonstrated that this method is effective and reliable for screening in both children and adults [15-18]. One of the major benefits of automated audiometry is its potential applications in situations or locations where audiologists or sound insulated booths are unavailable. Tablet devices present a unique platform to utilize the automated audiometric technology in a portable fashion.

A portable automated audiometer for the Apple iOS platform (SHOEBOX ${ }^{\mathrm{rm}}$ Audiometry, SHOEBOX Inc., Ottawa, ON), has previously been described and utilized in a variety of clinical scenarios [19-23]. This method of testing provides the tools to non-hearing healthcare experts to facilitate point of care evaluation, where the patient seeks the sound stimulus through an interactive and intuitive platform.

Previously published studies were completed on earlier versions of the software and did not evaluate the internal reliability or word recognition testing modality. Therefore, the objective of this multi-group study was to evaluate the capability of the tablet audiometer and record its performance when used in an adult patient population. In Group 1, the performance and the capability to measure hearing loss of the tablet audiometer was compared to conventional audiometry. In Group 2, word recognition testing using the tablet audiometer was compared against conventional audiometry. Finally, in Group 3 , the internal reliability of the tablet audiometer was evaluated using a test-retest methodology.

\section{Methodology}

This study was approved by The Ottawa Health Science Network Research Ethics Board (OHSN-REB \#20150561-01H). The tablet audiometer meets ANSI/ ASA S3.6-2010 requirements for audiometry, and is registered with Health Canada and the FDA as a Class II Medical Device for adults and children. Calibration of the tablet-audiometry transducers are completed every 12 months or sooner in accordance with the ANSI/ASA S3.6-2010 Specifications for Audiometers guidelines.

This study was administered in three separate groups of participants between May 2016 and October 2017. For all groups, adults over the age of 18 years who attended the Otolaryngology/Audiology clinic at The Ottawa Hospital were eligible to participate. Potential participants were excluded if they self-reported as being unable for any reason to use a tablet device or in Group 2 , anyone who had masking during word recognition testing in the sound booth. Group 2 participants were also limited to English speaking adults.

The Clinical Research Coordinator (CRC) was present to explain the study, answer questions and obtain informed consent. Eligible patients who provided consent were enrolled in the study. During the execution of the automated tablet audiometric testing, the CRC remained present to answer questions, observe behavior, and anecdotally record participant feedback while the participant completed the automated audiometric test. The CRC has a nursing background but has not received any formal audiological training.

Conventional audiometric evaluation was carried out by audiologists in a double walled sound booth using a GSI-61 Audiometer and followed standard protocols outlined in the "Practice Standards and Guidelines for Hearing Assessment of Adults by Audiologists," from the College of Audiologists and Speech-Language Pathologists of Ontario (CASLPO) [24].

In Groups 1 and 3, hearing testing with the tablet audiometer was performed; pure tone air conduction thresholds were obtained through the automated game platform. The software employs a modified HughsonWestlake protocol with a two-alternative force choice paradigm (i.e. participant sorts an object based on whether or not a sound is heard). All participants were tested at frequencies of 250, 500, 1000, 2000, 4000, and $8000 \mathrm{~Hz}$ using calibrated ER3A audiometric insert transducers. Lower and upper tone presentation volumes 
Table 1 - Demographic data for Group 1

\begin{tabular}{ll}
\hline Demographic characteristics & $N=40$ \\
\hline Age (years); mean (SD) & $54.7(18.4)$ \\
Age (years); median (range) & $53.8(20.3-86.9)$ \\
Female; $n$ (\%) & $24 / 40(60.0)$ \\
Proportion of participants with two ears assessed; $n(\%)$ & $39 / 40(97.5)$ \\
\hline
\end{tabular}

were limited at $10 \mathrm{~dB}$ and $90 \mathrm{~dB}$, respectively. Masking using the tablet audiometer occurs automatically in accordance with Katz et al. methods of clinical masking that have been previously described [25].

\section{Group 1}

Each participant underwent conventional pure tone audiometric testing administered by an audiologist followed by an automated tablet audiometric test. The tablet audiometric evaluations were performed in a quiet but non sound-insulated clinical exam room. Both tests were conducted using conventional ER3A inserts transducers on the same day.

\section{Group 2}

Participants underwent word recognition testing using both conventional audiometry as well as tablet audiometry. Word recognition was performed by way of the Northwestern University Auditory Test \# 6 (NU-6, Form A Lists 1-4, Auditec Inc). In view that the recorded NU-6 word list is available in English only, participants required a working understanding of English in order to participate in Group 2 of this study.

Patients first underwent conventional audiometry including word recognition testing performed by an Audiologist in a sound insulated booth. The Audiologist presented recorded NU-6 lists $1 \mathrm{a}$ and $1 \mathrm{~b}$ (50 words) using calibrated ER3A inserts at the patient's most comfortable listening level (MCL). Pre-recorded word lists were used rather than live voice during traditional audiometry to enhance standardization.

Next, the tablet audiometer was manually configured to perform the word recognition testing using the same MCL that was determined by the Audiologist during the patient audiogram performed in the sound booth. Word recognition testing by the tablet audiometer was performed using calibrated ER3A inserts in a non-sound- insulated clinical exam room. The word was presented to the patient through the insert transducers, the patient then repeated the word to the CRC who recorded the patient's accuracy on the tablet audiometer. During the tablet word recognition testing, NU-6 $2 \mathrm{a}$ and $2 \mathrm{~b}$ word lists (50 words) were used in order to minimize the risk of recall by the participants between the two tests.

\section{Group 3}

Participants self-administered the tablet audiogram twice in sequence using calibrated TDH-50 supra-aural headphones in a quiet, but non sound-insulated clinical exam room. The first test was performed and this was immediately followed by a second repeat tablet audiometric evaluation. The headphones were neither removed nor repositioned between tests.

\section{Statistical analysis \\ Group 1}

Clinically relevant hearing loss was, for the purposes of this analysis, defined as at least one threshold at or above $40 \mathrm{~dB}$ HL for either ear. As a sensitivity analysis, a threshold of $30 \mathrm{~dB}$ HL was also evaluated. 95\% confidence intervals for sensitivity and specificity were computed using the Wilson score method [26].

Agreement between traditional audiometry and tablet audiometry was evaluated using Cohen's unweighted kappa (for absolute agreement) and Cohen's weighted kappa (to take the extent of disagreement into account).

The percentage of agreements within $10 \mathrm{~dB}$ and within $5 \mathrm{~dB}$ were calculated with $95 \%$ Wilson-score confidence intervals. The mean percentage agreement across all frequencies was computed with $95 \%$ confidence intervals derived from Student's t-distribution. We conducted the analysis with and without frequencies of $250 \mathrm{~Hz}$ and $8000 \mathrm{~Hz}$ as measurements at these two frequencies have been known to be affected by background noise and transducer placement and tinnitus [27].

\section{Group 2}

To evaluate for significant differences in computed word recognition scores we used the critical difference range that has previously been well described for comparing consecutive word recognition testing [28]. The mean difference between tablet and conventional audiometric

Table 2 - Percentage agreement (within $10 \mathrm{~dB}$ ) between tablet audiometer and conventional audiometer at frequencies of $250 \mathrm{~Hz}$, $500 \mathrm{~Hz}, 1000,2000 \mathrm{~Hz}, 4000 \mathrm{~Hz}$, and $8000 \mathrm{~Hz}$

\begin{tabular}{lcccccccc}
\hline & $250 \mathrm{~Hz}$ & $500 \mathrm{~Hz}$ & $1000 \mathrm{~Hz}$ & $2000 \mathrm{~Hz}$ & $4000 \mathrm{~Hz}$ & $8000 \mathrm{~Hz}$ & mean & $\begin{array}{l}\text { mean excluding } \\
\text { AC250 Hz and AC8000 Hz }\end{array}$ \\
\hline Percent Agreement & 85.9 & 91.8 & 97.1 & 96.9 & 100.0 & 85.5 & 92.9 & 95.7 \\
95\% low & 76.0 & 83.2 & 89.9 & 89.5 & 94.0 & 74.7 & 88.8 & 92.6 \\
$95 \%$ High & 92.2 & 96.2 & 99.2 & 99.2 & 100.0 & 92.2 & 97.6 & 98.9 \\
\hline
\end{tabular}


Table 3 - Percentage agreement (within $5 \mathrm{~dB}$ ) between tablet audiometer and conventional audiometer at frequencies of $250 \mathrm{~Hz}$, $500 \mathrm{~Hz}, 1000,2000 \mathrm{~Hz}, 4000 \mathrm{~Hz}$, and $8000 \mathrm{~Hz}$

\begin{tabular}{lllllllll}
\hline & $250 \mathrm{~Hz}$ & $500 \mathrm{~Hz}$ & $1000 \mathrm{~Hz}$ & $2000 \mathrm{~Hz}$ & $4000 \mathrm{~Hz}$ & $8000 \mathrm{~Hz}$ & mean & mean excluding $250 \mathrm{~Hz}$ and $8000 \mathrm{~Hz}$ \\
\hline Percent Agreement & 61.6 & 75.7 & 85.7 & 90.9 & 93.4 & 65.1 & 78.7 & 84.9 \\
95\% low & 50.2 & 64.8 & 75.7 & 81.6 & 84.3 & 52.8 & 69.9 & 77.7 \\
95\% High & 71.9 & 84.0 & 92.1 & 95.8 & 97.4 & 75.7 & 89.2 & 92.1 \\
\hline
\end{tabular}

assessments for each patient was obtained to further characterize the performance of the tablet audiometer to perform word recognition testing. In their 1978 paper, Thornton and Raffin determined that with a 25 set word list there are wide ranges in percent scores that are in reality clinically irrelevant. These critical difference ranges narrow somewhat with a 50 word set. For the purposes of this study actual percent scores were classified using the critical difference ranges for both methods of testing. Percent agreement was determined along with 95\% confidence intervals.

\section{Group 3}

A mixed effects model was used to determine if the thresholds obtained with the two tablet tests were significantly different from each other. One-way intraclass correlation coefficient (ICC) was computed to determine the internal reliability. The mean difference between repeat assessments for each patient was obtained to further characterize the nature of the reliability. ICC was selected because - unlike Pearson correlation - it can detect systematic absolute differences between repeat assessments.

\section{Results}

\section{Group 1}

A total of 40 patients were included in this group. The mean age was 54.7 years old and $60 \%$ patients were female (Table 1). Hearing levels for patients in this group are detailed in Table 8.

When we compared the measured pure tone thresholds computed using the tablet audiometer to standard audiometry, we found that $92.9 \%$ of thresholds at all frequencies were within $10 \mathrm{~dB}$. When we excluded frequencies of $250 \mathrm{~Hz}$ and $8000 \mathrm{~Hz}, 95.7 \%$ of thresholds were

Table 4 - $2 \times 2$ contingency table comparison of tablet audiometry to conventional audiometry when hearing loss defined as at least one threshold of $40 \mathrm{~dB} \mathrm{HL}$ or greater in at least one ear

\begin{tabular}{lcc}
\hline & \multicolumn{2}{c}{ Traditional Audiometry } \\
\cline { 2 - 3 } Tablet audiometry (SHOEBOX) & Normal hearing & Hearing loss \\
\hline Normal hearing & 13 & 1 \\
Hearing loss & 0 & 25 \\
Sensitivity: 96\% (95\% Cl 81, 99\%), Specificity: & 100\% (95\% Cl 77, 100\%) \\
\hline
\end{tabular}

within $10 \mathrm{~dB}$ and $84.9 \%$ of thresholds were within $5 \mathrm{~dB}$ (Tables 2 and 3 ).

A sensitivity and specificity analysis are shown in Tables 4 and 5. When hearing loss was defined as having at least one threshold of $40 \mathrm{~dB} \mathrm{HL}$ or greater in at least one ear the sensitivity and specificity was calculated to be 96 and 100\% respectively (Table 4). When hearing loss was defined as having at least one threshold of $30 \mathrm{~dB}$ HL or greater in at least one ear the sensitivity was $100 \%$ with a specificity of $91 \%$ (Table 5).

\section{Group 2}

In Group 2 of this trial we recruited 44 patients. The average age was 55.2 and $68.2 \%$ of patient population was female (Table 6).

When we compared the word recognition scores using conventional audiometry and the tablet audiometer we determined that $96.2 \%$ (95\% CI 89.5, 98.7\%) of word recognition scores fell within the critical difference range and could therefore be considered equivalent. BlandAltman graph is depicted in Fig. 1.

\section{Group 3}

40 patients were enrolled in the sub-study. Two patients were excluded for declining to complete both tablet audiogram tests. The mean age of the patient population was 40 and $60.5 \%$ of patients were female (Table 7). Hearing levels for patients in this group are detailed in Table 8.

A correlation graph for test 1 and test 2 pure tone thresholds are shown in Fig. 2. The ICC for agreement between test 1 and test 2 for both left and right-ear pure tone thresholds was 0.98 .

Table 5 - $2 \times 2$ contingency table comparison of tablet audiometry to conventional audiometry when hearing loss defined as at least one threshold of $30 \mathrm{~dB} \mathrm{HL}$ or greater in at least one ear

\begin{tabular}{lcl}
\hline & \multicolumn{2}{c}{ Traditional Audiometry } \\
\cline { 2 - 3 } Tablet audiometry (SHOEBOX) & Normal hearing & Hearing loss \\
\hline Normal hearing & 10 & 0 \\
Hearing loss & 1 & 28 \\
Sensitivity: $100 \%$ (95\% Cl 88, 100\%), Specificity: $91 \%(95 \% \mathrm{Cl} 62,98 \%)$ \\
\hline
\end{tabular}


Table 6 - Demographic data for Group 2

\begin{tabular}{ll}
\hline Demographic characteristics & $N=44$ \\
\hline Age, mean (SD) & $55.2(14.8)$ \\
Age, median (IQR) & $55.6(44.7,64.9)$ \\
Female, $n(\%)$ & $30(68.2)$ \\
participants with two ears assessed, $n(\%)$ & $39(88.6)$ \\
\hline
\end{tabular}

For the left ear, the mean difference between repeat assessments was $0(\mathrm{SD}=2.1)$. The $95 \%$ limits of agreement were $-4.1 \mathrm{~dB}(95 \% \mathrm{CI}-5.2,-2.9)$ to $4.1 \mathrm{~dB}(95 \%$ CI 2.9, 5.2). On the right side, the mean difference was 0.1 ( $\mathrm{SD}=1.1)$. The $95 \%$ limits of agreement were $-2 \mathrm{~dB}$ (95\% CI $-2.6,-1.4)$ to $2.1 \mathrm{~dB}(95 \%$ CI 1.5, 2.8) (Fig. 3).

\section{Discussion}

In this multi-cohort prospective study three adult cohorts underwent testing using the tablet audiometer. In Group 1, we compared the tablet audiometer to conventional audiometry. There was overall high degree of agreement between both modalities with 97\% of thresholds measured within $10 \mathrm{~dB}$. The impact of ambient noise in the accuracy of threshold testing has previously been described and most affects thresholds measured at $250 \mathrm{~Hz}$ and $8000 \mathrm{~Hz}[19,27,29]$. Although the ambient background noise was not recorded for this study, testing was performed in a quiet clinical exam room with adjacent rooms occupied by patients attending Otolaryngology clinic. Overall, our study suggests that the tablet audiometer reported similar threshold values as traditional audiometry despite testing being performed in a nonsound insulated booth and without the coaching of a trained Audiologist. In two circumstances there was a discordance between the audiologist and tablet audiometer as to when to apply masking. The tablet audiometer is programmed to apply masking in a rule based fashion in accordance with standard audiometric practices [25]. The audiologist, in two circumstances, applied masking based on their expert opinion and experience with consideration for individual patient factors. In these scenarios the masked value was compared to the unmasked tablet audiometric threshold. Despite a discordance in the application of masking between test conditions the results suggest a high degree of agreement between tablet and conventional audiometric threshold measurements.

The definition of hearing loss that was used was meant to reflect a clinically significant hearing loss whereby a clinician may decide to offer the patient some form of intervention. We chose to characterize hearing loss by the worse ear to represent the limitations of the patient's unaided hearing ability. As such, we used to Clinically this provides clinicians with the ability to counsel patients about how their hearing may be impacting their ability to communicate. We performed a sensitivity analysis using both $30 \mathrm{~dB}$ and $40 \mathrm{~dB} \mathrm{HL}$ as the definition of hearing loss to enable comparisons to previous studies that have performed sensitivity and specificity analyses [19-21]. Overall, the calculated sensitivity and specificity

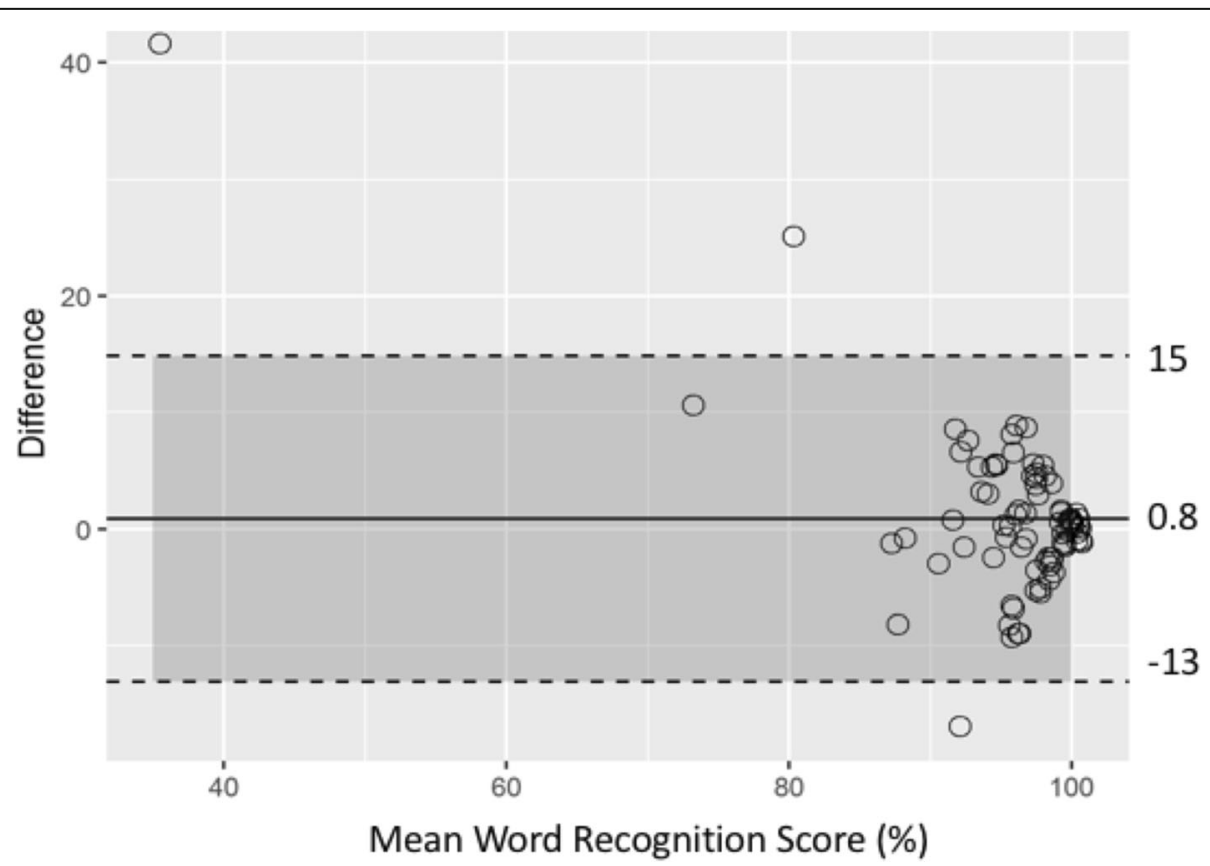

Fig. 1 Group 2 Bland Altman Plot - Inter-score difference for word recognition scores between tablet and conventional assessments. Dotted lines depict $95 \%$ limits of agreement. Mean difference of 0.8 (95\% Cl 15,-13) 
Table 7 - Demographic data for Group 3

\begin{tabular}{ll}
\hline Demographic characteristics & $N=38$ \\
\hline Age (years); mean (SD) & $39.4(15.9)$ \\
Age (years); median (range) & $36.8(19.2-73.8)$ \\
Female; $n$ (\%) & $23(60.5)$ \\
Proportion of participants with two ears assessed; $\mathrm{n}(\%)$ & $37(97.4)$ \\
\hline
\end{tabular}

values remained comparable. When the definition of hearing loss was lowered to $30 \mathrm{~dB}$ HL the sensitivity improved and the specificity decrease slightly.

Overall our results in Group 1 are similar to previous studies which have compared the tablet audiometer to conventional audiometry [19-22]. In previous studies that have evaluated the performance of other automated devices, $86-95 \%$ of tested thresholds have been shown to be within $10 \mathrm{~dB}$ or less compared to conventional audiometry [18, 29-32]. Thus, our results would suggest that the tablet audiometer performs at least similarly to other automated devices. Post hoc analysis revealed an important discrepancy between automated and standard audiometric methods of testing. Specifically, the automated test was set with a lower limit of $10 \mathrm{~dB}$ and an upper limit of $90 \mathrm{~dB}$. Whereas, the clinical protocol that was used to perform conventional audiometric testing was to perform hearing threshold testing to $-10 \mathrm{~dB}$. Hence, inherent differences were created by the methodology in certain instances, as a result, only frequencies where comparisons were possible for these patients were included in the statistical analyses. Future studies could mitigate this risk by aligning the protocols for tablet and traditional audiometric testing. Two additional changes were made post-hoc to our statistical analyses. As seen in Tables 2 and 3, we performed our statistical analysis for all frequencies measured as well as excluding 250 and $8000 \mathrm{~Hz}$. There are several factors that may alter threshold measurements at these frequencies including ambient noise, transducer placement and tinnitus [19, 27, 29]. As a result threshold measurements at $250 \mathrm{~Hz}$ and $8000 \mathrm{~Hz}$ were removed to better represent the environment in which patients were being tested.
In Group 2, we examined the performance of the tablet audiometer to perform word recognition testing. Overall, $96.2 \%$ of scores recorded were considered equivalent. The majority $(n=43)$ of patients were found to have word recognition scores $\geq 80 \%$. The high scores are expected given that the testing is completed at an optimal listening level for patients. The generalizability of the results to patients with lower word recognition scores is not clear.

This is the first study that has examined word recognition testing using the tablet audiometer. The NU-6 word list was used in both the conventional and audiometric testing. Since the tablet audiometer is an automated device the NU-6 word list was pre-recorded and played to the patient through the tablet device. Given that the word list was pre-recorded there was no opportunity to repeat the test word back to the patient in circumstances where the word was not heard due to background noise, technical issues or any other interference. Furthermore, the NU-6 word list is only available in English, monosyllabic word lists would need to be added to the software platform to facilitate inclusion patients who speak other languages.

In order to accurately compare the performance of the tablet audiometer to perform word recognition testing, the MCL established and used in the sound booth by an Audiologist was also used for the tablet audiometer testing. Additionally, the MCL should be established by someone experienced with hearing testing and the process for establishing such levels. Because the MCL is supra-threshold, it is less impacted by ambient noise therefore using the same MCL for the two test modalities is appropriate. The tablet audiometer software does allow test administrators to establish MCL for patients for general clinical use.

In Group 3, the internal reliability of the tablet audiometer was tested using a test-retest cohort design. The ICC, a measurement of internal reliability, was exceptionally high at 0.98 . Moreover, the mean difference between assessments is low with narrow variance at $0 \mathrm{~dB}$ $(\mathrm{SD}=2.1 \mathrm{~dB})$ and $0.1 \mathrm{~dB}(\mathrm{SD}=1.1 \mathrm{~dB})$ in left and right ears respectively, suggesting that there is minimal

Table 8 - Hearing levels of participants in Groups 1 and 3. Hearing status based on the worst frequency (500, 1000. 2000, 4000 Hz) in the worse ear

\begin{tabular}{llll}
\hline Hearing Category & Range & $\begin{array}{l}\text { Group 1 } \\
\text { (number of participants) }\end{array}$ & $\begin{array}{l}\text { Group 3 } \\
\text { (number of participants) }\end{array}$ \\
\hline Normal & $\leq 25 \mathrm{~dB} \mathrm{HL}$ & 11 & 26 \\
Mild & $26-40 \mathrm{~dB} \mathrm{HL}$ & 5 & 9 \\
Moderate & $41-55 \mathrm{~dB} \mathrm{HL}$ & 12 & 2 \\
Moderately Severe & $56-70 \mathrm{~dB} \mathrm{HL}$ & 5 & 0 \\
Severe & $71-90 \mathrm{~dB} \mathrm{HL}$ & 5 & 1 \\
Profound & $\geq 91 \mathrm{~dB} \mathrm{HL}$ & 2 & 0 \\
\hline
\end{tabular}



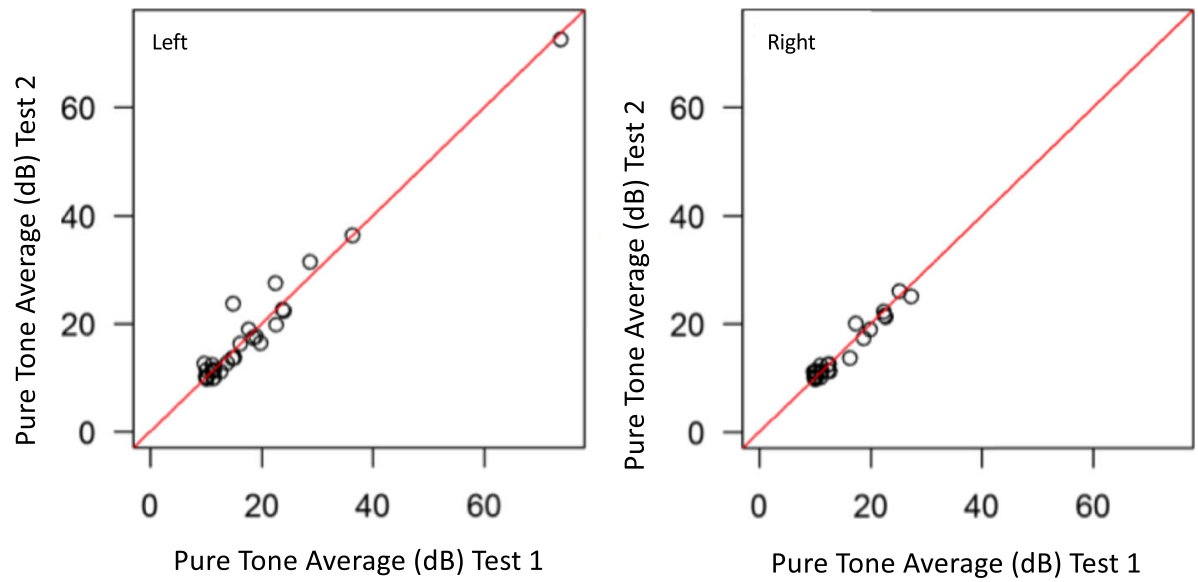

Fig. 2 Group 3 Pure-tone threshold correlation graph for test 1 and test 2. ICC for agreement in both the left and right is 0.98

clinical impact in any variances between repeat assessments. A larger 95\% confidence range existed for the left ear. We feel this likely reflects a learning effect given that the left ear was programmed to be tested first followed by the right ear, resulting in diminished variability for the right ear. Nonetheless, for both the left and right ear, the $95 \%$ confidence intervals were within $10 \mathrm{db}$ when using the portable audiometer. This is comparable to previous studies which have examined the internal reliability of conventional audiometry in healthy subjects [33].

A potential source of bias that was identified for all cohorts is the possible result of a learning effect as patients gain experience and learn the skills required to perform accurately on both conventional and tablet audiometry. Future studies could mitigate this bias by randomizing the order in which the tablet and conventional audiometric evaluations are performed.
In our opinion, the implementation of a portable selfadministered tablet audiometer as a screening tool has the potential to reduce the number of referrals for conventional audiometry. Ideally, this could lead to a significant reduction in public health care costs and audiology wait times. In addition, the tablet audiometer could improve access to screening audiology, in particular, for vulnerable patient populations where access to conventional audiometry is scarce. Future studies using the tablet audiometer should aim to investigate the performance of the tablet audiometer in other clinical scenarios or patient populations such as cochlear implant transducer calibration, primary care facilities as well as tertiary care capacities, monitoring for ototoxicity in intensive-care, or oncology units. Future research is required to compare bone conduction testing as well as speech reception threshold testing between the sound booth and tablet-based audiometer.
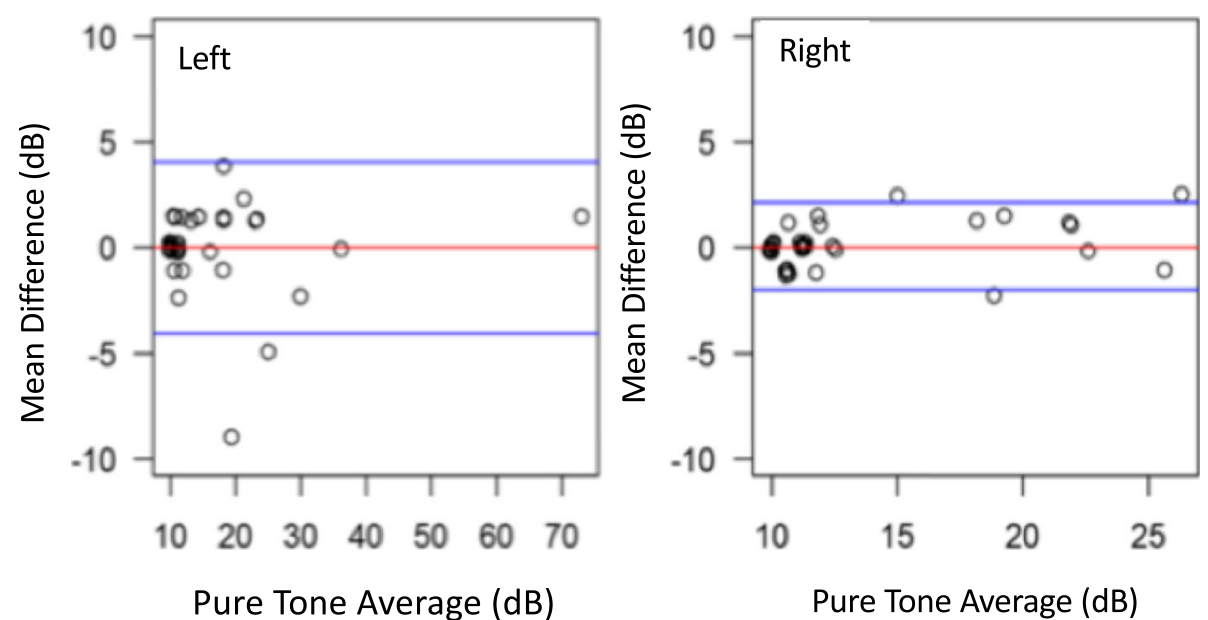

Pure Tone Average $(\mathrm{dB})$

Fig. 3 Group 3 Bland Altman Plot - Mean differences in pure tone thresholds between repeat assessments. Blue lines depict 95\% limits of agreement. Mean difference of $0(S D=2.1)$ on the left, and $0.1(S D=1.1)$ on the right 


\section{Conclusions}

The objective of this study was to examine the validity and internal reliability of the tablet audiometer and secondarily evaluate the performance of the tablet audiometer to perform word recognition scoring. The results of this study indicate that adult audiometry and word recognition testing appears valid when performed by nonhealthcare experts using a tablet audiometer outside of a sound booth in a quiet environment.

\section{Abbreviations}

ANSI: American National Standards Institute; CASLPO: College of Audiologists and Speech-Language Pathologists of Ontario; CRC: Clinical Research Coordinator; dB HL: decibels Hearing Level; dB: Decibel; FDA: Food and Drug Administration; ICC: Intra-class correlation coefficient; MCL: Most comfortable level; NU-6: Northwestern University Word list

\section{Acknowledgments}

Special thanks to the Clinical Research Coordinator Ms. Debora Hogan, RN. In addition, the Audiologists at The Ottawa Hospital including Megan Scott, Leanne Bowden and Jennifer Platt-Talbot who helped facilitate patient participation and audiometric testing and Nick Barrowman for his statistical analysis.

\section{Author contributions}

$\mathrm{MB}$ contributed to the conception of research project, research design and drafting the manuscript. AEM contributed to the acquisition, analysis, interpretation of the data and revising the manuscript. AM contributed to research design and plan, acquisition, analysis and drafting the manuscript. DS contributed to the research design. RL contributed to the conception of the research and the design and interpretation of the results. MB (Bromwhich) is the corresponding author and contributed to the conception of research project, research design and plan, acquisition analysis, drafting manuscript. All authors read and approved the final manuscript.

\section{Funding}

Not applicable.

\section{Ethics approval and consent to participate}

This study was approved by The Ottawa Health Science Network Research Ethics Board (OHSN-REB \#20150561-01H). Consent to participate in this study was obtained from each individual participant.

\section{Consent for publication}

Availability of data and material: The datasets generated and/or analysed during the current study are not publicly available due to patient privacy concerns but are available from the corresponding author on reasonable request.

\section{Competing interests}

Dr. Matthew Bromwich is Co-Founder and Chief Medical Officer SHOEBOX Inc. Amy E Mark holds a joint Clinical Research Associate position with SHOEBOX Inc. and the Children's Hospital of Eastern Ontario. Renée Lefrançois is the Director of Audiology at SHOEBOX Inc.

\section{Author details}

${ }^{1}$ Faculty of Medicine, University of Ottawa, Ottawa, Ontario, Canada. ${ }^{2}$ Department of Otolaryngology - Head and Neck Surgery, The Ottawa Hospital, Ottawa, Ontario, Canada. ${ }^{3}$ Department of Otolaryngology - Head and Neck Surgery, University of Ottawa, Ottawa, Ontario, Canada. ${ }^{4}$ Division of Pediatric Otolaryngology - Head and Neck Surgery, Children's Hospital of Eastern Ontario, Ottawa, Ontario, Canada. ${ }^{5}$ Children's Hospital of Easter Ontario Research Institute, Ottawa, Canada. ${ }^{6}$ SHOEBOX Inc, Ottawa, Ontario, Canada. ${ }^{7}$ Ottawa Hospital Research Institute, Ottawa, Ontario, Canada.
Received: 9 April 2018 Accepted: 22 October 2019

Published online: 07 November 2019

\section{References}

1. Statistics Canada. Canadian Health Measures Survey: Household and Physical Measures Data, 2012 to 2013: Body Mass Index of Children and Youth.; 2014. http://www.statcan.gc.ca/pub/82-625-x/2014001/article/14105eng.htm.

2. Statistics Canada. Population Projections for Canada, Provinces and Territories: 2013-2036.; 2014. doi:91-520-X.

3. Arlinger $\mathrm{S}$. Negative consequences of uncorrected hearing loss - a review. Int J Audiol. 2003;42(2):S17-20. https://doi.org/10.3109/14992020309074639.

4. Plack CJ, Barker D, Prendergast G. Perceptual consequences of "hidden" hearing loss. Trends Hear. 2014;18. https://doi.org/10.1177/ 2331216514550621.

5. Ciorba A, Pelucchi S, Pastore A. The impact of hearing loss on the quality of life in adults. Srp Arh Celok Lek. 2012;139(5-6):286-90. https://doi.org/10. 2147/cia.s26059

6. Dawes P, Emsley R, Cruickshanks KJ, et al. Hearing loss and cognition: The role of hearing aids, social isolation and depression. PLoS One. 2015;10(3). https://doi.org/10.1371/journal.pone.0119616.

7. Cornwell EY, Waite $\amalg$. Social disconnectedness, perceived isolation, and health among older adults. J Health Soc Behav. 2009;50(1):31-48. https:// doi.org/10.1177/002214650905000103.

8. Mick P, Kawachi I, Lin FR. The association between hearing loss and social isolation in older adults. Otolaryngol - Head Neck Surg (United States). 2014; 150(3):378-84. https://doi.org/10.1177/0194599813518021.

9. Niemensivu R, Manchaiah V, Roine RP, Kentala E, Sintonen H. Health-related quality of life in adults with hearing impairment before and after hearingaid rehabilitation in Finland. Int J Audiol. 2015;54(12):967-75. https://doi.org/ 10.3109/14992027.2015.1077400.

10. Vuorialho A, Karinen P, Sorri M. Effect of hearing aids on hearing disability and quality of life in the elderly. Int J Audiol. 2006;45(7):400-5. https://doi. org/10.1080/14992020600625007

11. Brennan-Jones CG, Taljaard DS, Brennan-Jones SEF, Bennett RJ, Swanepoel DW, Eikelboom RH. Self-reported hearing loss and manual audiometry: a rural versus urban comparison. Aust J Rural Health. 2016;24(2):130-5. https:// doi.org/10.1111/ajr.12227.

12. Windmill IM, Freeman BA. Demand for audiology services: $30-Y r$ projections and impact on academic programs. J Am Acad Audiol. 2013;24(5):407-16. https://doi.org/10.3766/jaaa.24.5.7.

13. Sokol J. Hearing Screening. doi:https://doi.org/10.1080/10904018.2012. 677670

14. Crowson MG, Schulz K, Tucci DL. Access to health care and hearing evaluation in US adults. Ann Otol Rhinol Laryngol. 2016;125(9):716-21. https://doi.org/10.1177/0003489416649972.

15. Foulad A, Bui P, Djalilian H. Automated audiometry using apple iOS-based application technology. Otolaryngol - Head Neck Surg (United States). 2013; 149(5):700-6. https://doi.org/10.1177/0194599813501461.

16. Eikelboom RH, Swanepoel DW, Motakef S, Upson GS. Clinical validation of the AMTAS automated audiometer. Int J Audiol. 2013;52(5):342-9. https:// doi.org/10.3109/14992027.2013.769065.

17. Derin S, Cam OH, Beydilli H, Acar E, Elicora SS, Sahan M. Initial assessment of hearing loss using a mobile application for audiological evaluation. J Laryngol Otol. 2016;130(3):248-51. https://doi.org/10.1017/ S0022215116000062.

18. Brennan-Jones CG, Eikelboom RH, Swanepoel DW, Friedland PL, Atlas MD. Clinical validation of automated audiometry with continuous noisemonitoring in a clinically heterogeneous population outside a soundtreated environment. Int J Audiol. 2016;55(9):507-13. https://doi.org/10. 1080/14992027.2016.1178858.

19. Saliba J, Al-Reefi M, Carriere JS, Verma N, Provencal C, Rappaport JM. Accuracy of Mobile-based audiometry in the evaluation of hearing loss in quiet and Noisy environments. Otolaryngol - Head Neck Surg (United States). 2017;156(4):706-11. https://doi.org/10.1177/0194599816683663.

20. Thompson GP, Sladen DP, Borst BJH, Still OL. Accuracy of a tablet audiometer for measuring behavioral hearing thresholds in a clinical population. Otolaryngol - Head Neck Surg (United States). 2015;153(5):83842. https://doi.org/10.1177/0194599815593737.

21. Yeung J, Javidnia H, Heley S, Beauregard Y, Champagne S, Bromwich M. The new age of play audiometry: prospective validation testing of an iPad- 
based play audiometer. J Otolaryngol Head Neck Surg. 2013;42:21. https:// doi.org/10.1186/1916-0216-42-21.

22. Yeung JC, Heley S, Beauregard Y, Champagne S, Bromwich MA. Selfadministered hearing loss screening using an interactive, tablet play audiometer with ear bud headphones. Int J Pediatr Otorhinolaryngol. 2015; 79(8):1248-52. https://doi.org/10.1016/j.jpporl.2015.05.021.

23. Rourke R, Kong DCC, Bromwich M. Tablet audiometry in Canada's north: a portable and efficient method for hearing screening. Otolaryngol - Head Neck Surg (United States). 2016;155(3):473-8. https://doi.org/10.1177/ 0194599816644407.

24. College of Audiologists and Speech Language Pathologists of Ontario C. Practice Standards and Guidelines for the Assessment of Children by Speech-Language Pathologists. 2008. http:/www.caslpo.com/sites/default/ uploads/files/PSG_EN_Hearing_Assessment_of_Adults_by_Audiologists.pdf.

25. Katz J, Chasin M, English K, Hood L, Tillery K. Handbook of Clinical Audiology.; 2015. doi:https://doi.org/10.1111/j.1365-313X.2005.02390.X.

26. Wilson EB. Probable inference, the law of succession, and statistical inference. J Am Stat Assoc. 1927;22(158):209-12. https://doi.org/10.1080/ 01621459.1927 .10502953

27. Paquier $M$, Koehl V, Jantzem B. Influence of headphone position in puretone audiometry. Presented at the: 2012.

28. Thornton AR, Raffin MJM. Speech-discrimination scores modeled as a binomial variable. J Speech, Lang Hear Res. 1978;21(3):507-18.

29. Storey KK, Muñoz K, Nelson L, Larsen J, White K. Ambient noise impact on accuracy of automated hearing assessment. Int J Audiol. 2014;53(10):730-6. https://doi.org/10.3109/14992027.2014.920110

30. Yu J, Ostevik A, Hodgetts B, Ho A. Automated hearing tests: applying the otogram to patients who are difficult to test. J Otolaryngol - Head Neck Surg. 2011;40(5):376-83. https://doi.org/10.2310/7070.2011.100095.

31. Ho ATP, Hildreth AJ, Lindsey L. Computer-assisted audiometry versus manual audiometry. Otol Neurotol. 2009;30(7):876-83. https://doi.org/10 1097/MAO.0b013e3181b120d0.

32. Swanepoel DW, Matthysen C, Eikelboom RH, Clark JL, Hall JW. Pure-tone audiometry outside a sound booth using earphone attentuation, integrated noise monitoring, and automation. Int J Audiol. 2015;54(11):777-85. https:// doi.org/10.3109/14992027.2015.1072647.

33. Schmuziger N, Probst R, Smurzynski J. Test-retest reliability of pure-tone thresholds from 0.5 to $16 \mathrm{kHz}$ using Sennheiser HDA 200 and Etymotic research ER-2 earphones. Ear Hear. 2004;25(2):127-32. https://doi.org/10. 1097/01.AUD.0000120361.87401.C8

\section{Publisher's Note}

Springer Nature remains neutral with regard to jurisdictional claims in published maps and institutional affiliations.

Ready to submit your research? Choose BMC and benefit from:

- fast, convenient online submission

- thorough peer review by experienced researchers in your field

- rapid publication on acceptance

- support for research data, including large and complex data types

- gold Open Access which fosters wider collaboration and increased citations

- maximum visibility for your research: over $100 \mathrm{M}$ website views per year

At BMC, research is always in progress.

Learn more biomedcentral.com/submissions 